\title{
Psycho-social factors associated with high depressive symptomatology in female adolescents and gender difference in adolescent depression: an epidemiological survey in China's Hubei Province
}

Wenzhe Sun', Junhua Mei², Yanyan Wang', Xin Zhao', Zhou Zhu'', Chenyan Zhang ${ }^{1}$, Chensheng Pan', Guo Li', Yuxi Chen ${ }^{3}$, Jinfeng Miao', Yan Lan', Xiuli Qiu ${ }^{1}$ and Yi Xü ${ }^{4 *}$

\begin{abstract}
Background: Exploring etiological clues to adolescent depression, especially in female adolescents, might be helpful to improve the social environment of female adolescents. The aim at this study is to explore psycho-social factors of female adolescents with high depressive symptomatology and gender differences in depressive symptoms among Chinese adolescents.

Method: We examined 4100 adolescents from Wuhan city and Jianli county via a cross-sectional study. Depressive symptomatology was screened through the Chinese version of Center for Epidemiology Studies Depression Scale. Multivariate logistic regression was performed to explore the factors related to high depressive symptomatology in female and male adolescents, respectively.

Results: The prevalence of high depressive symptomatology in female and male were 38.9 and $30.2 \%$ respectively. The psycho-social factors of high depressive symptomatology in female adolescents were age (Adjusted odds ratio $[\mathrm{aOR}]=1.201,95 \%$ confidence interval $[\mathrm{Cl}], 1.076 \sim 1.341$ ), single parent family (aOR $=2.004,95 \% \mathrm{Cl}, 1.448 \sim 2.772$ ) and fathers' education level (compared to primary school and below, [Junior middle school, aOR $=0.641,95 \% \mathrm{Cl}$, $0.439 \sim 0.934$; Senior middle school, $\mathrm{aOR}=0.603,95 \% \mathrm{Cl}, 0.410 \sim 0.888$; College degree and above, aOR $=0.639$, $95 \% \mathrm{Cl}, 0.437 \sim 0.936])$.
\end{abstract}

Conclusion: Fathers' education level was associated with high depressive symptomatology in female adolescents. Female adolescents whose father with primary school education or below deserves more attention. Further epidemiologic researches need to be conducted to explore the different risk factors between female and male adolescents in China.

Keywords: Female, Adolescents, Gender difference, Depressive symptomatology, Psycho-social factors

\footnotetext{
*Correspondence: xuyitj@hust.edu.cn

${ }^{4}$ Department of Plastic surgery, Tongji Hospital, Tongji Medical College,

Huazhong University of Science and Technology, No.1095 Jiefang Avenue, Wuhan 430030, China

Full list of author information is available at the end of the article
}

(c) The Author(s). 2021 Open Access This article is licensed under a Creative Commons Attribution 4.0 International License, which permits use, sharing, adaptation, distribution and reproduction in any medium or format, as long as you give appropriate credit to the original author(s) and the source, provide a link to the Creative Commons licence, and indicate if changes were made. The images or other third party material in this article are included in the article's Creative Commons licence, unless indicated otherwise in a credit line to the material. If material is not included in the article's Creative Commons licence and your intended use is not permitted by statutory regulation or exceeds the permitted use, you will need to obtain permission directly from the copyright holder. To view a copy of this licence, visit http://creativecommons.org/licenses/by/4.0/. The Creative Commons Public Domain Dedication waiver (http://creativecommons.org/publicdomain/zero/1.0/) applies to the data made available in this article, unless otherwise stated in a credit line to the data. 


\section{Introduction}

For the past few years, depression have become a major public health problem worldwide [1]. According to the World Health Organization (WHO), depression will be the leading cause of disease burden by 2030 [2]. In China, the new update from the National Health and Health Commission (2018) shows that about 30 million children and adolescents under the age of 17 are suffering from psychological disorders in China [3], while psychological disorders such as depression, anxiety and bipolar disorder have been neglected for a long time in China. Given that quite a few depression diagnoses have their initial origins in adolescence, it is important to identify depression and prevent the establishment of maladaptive cognitive or behavioral patterns among early adolescence [4-6].

The sociodemographic characteristics of China may play a prominent role in the occurrence of depressive symptoms in Chinese adolescents, since adolescents are more vulnerable to the surrounding environment and the individual psychological factors [7-10]. The situation of female adolescents in China is even more difficult. Gender factors have already been widely discussed and a considerable number of studies suggested that females, both adults and adolescents, have higher risk of depression than males $[11,12]$. Furthermore, owing to entrenched traditional social roles, son preference has always been a common problem in China. In recent years, early marriage and procreation rate of women rise again, although they dropped between 1990 and 2015 [13], which suggested that the educational inequities in female adolescents of China has recently exacerbated. However, few researchers have explored the different sociodemographic related factors between female adolescents and male adolescents.

Hence, it's necessary to conduct an exhaustive study about gender difference of psycho-social factors in China to provide clues for early intervention of adolescent depression. Furthermore, Hubei province, the place targeted in this study, had the highest rates of suicide in China [14], so it is appropriate for depression research. The objective of this study is to explore psycho-social factors of female adolescents with high depressive symptomatology and gender differences in depressive symptoms among Chinese adolescents.

\section{Method}

\section{Participants}

This epidemiological survey was conducted from November 2018 to February 2019 in Wuhan city and Jianli country of Hubei province, to define the prevalence and associated factors for high depressive symptomatology in female and male adolescents. This cross-sectional study was approved by our Institutional Review Board and participants were recruited for the project with approval from the school officials, and with approval from their guardians in written consent with the assistance of teachers. Three junior middle schools of Wuhan city and four junior middle schools of Jianli county were selected with stratified-cluster sampling method. The inclusion criteria for this study were: (1) adolescents in grades 7 to 9; (2) adolescents who were willing to take part in the survey. Individuals were excluded if any of the following criteria was met: (1) adolescents were unwilling to take part in the survey; (2) Adolescents did not complete the questionnaire. Eventually, 4,122 students were approached, 22 students declined to participate or incomplete the questionnaires, 4,100 junior high school students covering three grades aged from 11 to 16 years old were involved in analysis.

\section{Data collection}

The investigation was organized and coordinated by Department of Neurology, Tongji Hospital, Tongji Medical College, Huazhong University of Science and Technology. The investigators who conducted the questionnaire survey were trained uniformly. The survey was conducted through teachers before COVID, all data were collected via a paper demographic survey handed out in class by the adolescents themselves. The senior investigators checked the collected questionnaires daily to perform quality control. Data were entered double-blindly into the database by two different researchers using Epidata 3.0 to guarantee accuracy. All the data were collected through paper questionnaires, each of which contained an informed consent form filled out by the subject's parents or guardians.

\section{Outcomes}

Depressive symptomatology was screened through the Chinese version of Center for Epidemiology Studies Depression Scale (CES-D Scale). A score of 20 and above indicated high depressive symptomatology [15-19]. CES-D has been widely used in Chinese adolescents, with good reliability and validity $[20,21]$. The Cronbach's $\alpha$ coefficients of the CES-D Scale in this study were 0.87 .

\section{Variables}

The demographic characteristics were collected, consisting of rural area students, age, gender, boarding status, class status, household characteristics, single parent family, current caregivers, sibling child status, left behind types, father's and mother's education level, physical activity (PA), birth order and having siblings or not. The children are considered as the left-behind children (LBC) if his/her mother or father has left the local place for a job over the past 5 months. The left behind 
children were further divided into three subgroups: father absent, mother absent and both absent. Physical activity (PA) were assessed by Global Physical Activity Questionnaire, which were defined as up to the WHO physical activity standards that children and youth aged 5-17 years old should accumulate at least $60 \mathrm{~min}$ of moderate- to vigorous-intensity physical activity daily, most of the daily physical activity should be aerobic, and vigorous-intensity activities should be incorporated, including those that strengthen muscle and bone, at least 3 times per week.

\section{Statistical analyses}

Statistical analyses to identify related factors were performed using SPSS software version 22.0 (Statistical Package for the Social Sciences) for Windows (SPSS, Chicago, IL). Continuous data were presented as the medians and interquartile ranges (IQR) and compared using Mann Whitney $U$ test. Categorical variables are presented as frequencies (proportions) and compared with Chi-square test or Fisher's exact test. The univariate analyses in male and female subgroups were using univariate logistic regression. Variables with a $P$ value less than 0.1 in univariate analyses were subjected to multivariable logistic regression analysis. Multivariate logistic regression was performed to explore the factors related to high depressive symptomatology and calculate the adjusted odds ratios (ORs) and 95\% confidence intervals (CIs). All $P$-values were two-sided, and $P<0.05$ was considered statistically significant.

\section{Results}

\section{Sample characteristics}

Table 1 presents comparison of social characteristics of the adolescents with high depressive symptomatology and low depressive symptomatology and factors associated with high depressive symptomatology. In this sample, the age distribution was 11 to 16 years. The total prevalence of high depressive symptomatology was $34.0 \%$. The adolescents with high depressive symptomatology were older $(p<0.001)$, more female $(p<0.001)$, more key class students $(p=0.004)$, getting less physical activity $(p<0.001)$ and more likely from single parent family $(p<0.001)$. All variables with $p<0.1$ in univariate analysis were included in multivariable logistic regression models for adjustment (Table 1). After multivariate logistic regression analysis, we found that age $(\mathrm{aOR}=1.150,95 \% \mathrm{CI}, 1.062 \sim 1.260, p=0.001)$, female $(\mathrm{aOR}=1.444,95 \% \mathrm{CI}, 1.260 \sim 1.655, p<0.001)$, single parent family $(\mathrm{aOR}=1.870,95 \% \mathrm{CI}, 1.524 \sim 2.294$, $p<0.001)$ and physical activity $(\mathrm{aOR}=0.678,95 \% \mathrm{CI}$, $0.572 \sim 0.804, p<0.001$ ) were independently associated with high depressive symptomatology.
Factors associated with high depressive symptomatology among female and male samples

We also compared the psycho-social characteristics of the female and male adolescents (Table 2). The prevalence of high depressive symptomatology in female and male were 38.9 and $30.2 \%$ respectively. The adolescents in female group were more key class students $(p<0.001)$, more raised by parents $(p=0.001)$, with higher fathers' and mothers' education background $(p<0.001)$, more likely to be the oldest children $(p<0.001)$ and have younger brother in family $(p<0.001)$. On the other hand, adolescents in female group were less rural students $(p<0.001)$, less from single parent family $(p=0.034)$, less likely to have physical activity $(p<0.001)$, less likely to live in school $(p<0.001)$ and have older sister in family $(p<0.001)$.

Furthermore, logistic regression was used to identify factors associated with high depressive symptomatology in female and male adolescents respectively (Table $3 \& 4$ ). The related factors of high depressive symptomatology in female adolescents were age $(\mathrm{aOR}=1.20195 \% \mathrm{CI}$, $1.076 \sim 1.341)$, single parent family $(\mathrm{aOR}=2.004,95 \% \mathrm{CI}$, $1.448 \sim 2.772$ ) and fathers' education level (compared to primary school and below, [Junior middle school, aOR = 0.641, 95\%CI, 0.439 0.934; Senior middle school, $\mathrm{aOR}=0.603,95 \% \mathrm{CI}, 0.410 \sim 0.888$; College degree and above, aOR $=0.639,95 \% \mathrm{CI}, 0.437 \sim 0.936]$ ) (Table 4). The factors independently associated with high depressive symptomatology in male adolescents were age, single parent family and physical activity.

\section{Discussion}

In this study, we aimed to explore psycho-social factors of female adolescents with high depressive symptomatology and gender differences in depressive symptoms among Hubei province. The total prevalence of this study was $34.0 \%$, it's similar to other areas in East Asia, including Shenzhen (34.7\%), Taiwan (30.2\%) and Korea (31.4\%) [22-24].

It is a unique phenomenon in China, especially in China's urban areas, that middle school students were divided into key classes and non-key classes according to their academic achievement [25]. Key classes mean "better students", "better teachers" and more high-quality educational resources, and adolescents in non-key class may experience discrimination. In this study, we found that compared to low depressive symptomatology, high depressive symptomatology group have more key class students (46.3\% VS. 41.6\%). However, after adjusting for confounding variables, key class was not a significantly associated factor for presenting with high depressive symptomatology.

New research from the UK is suggesting that higher birth order (later born) children were at increased risk of 
Table 1 Related factors of adolescent high depressive symptomatology in Hubei province

\begin{tabular}{|c|c|c|c|c|c|}
\hline \multirow[t]{2}{*}{ Correlates } & \multicolumn{3}{|l|}{ Univariate analysis } & \multicolumn{2}{|l|}{ Multivariate analysis } \\
\hline & $\begin{array}{l}\text { High depressive } \\
\text { symptomatology }(N=1393)\end{array}$ & $\begin{array}{l}\text { Low Depressive } \\
\text { symptomatology }(N=2707)\end{array}$ & $P$ & Adjusted OR (95\%Cl) & $P$ \\
\hline Rural areas, N (\%) & $626(44.9)$ & $1298(47.9)$ & 0.067 & $1.065(0.900 \sim 1.259)$ & 0.464 \\
\hline Age, y (IQR) & $14(13 \sim 14)$ & $13(13 \sim 14)$ & $<0.001^{*}$ & $1.150(1.062 \sim 1.260)$ & $0.001^{*}$ \\
\hline Female, N (\%) & $690(49.5)$ & $1084(40.0)$ & $<0.001^{*}$ & $1.444(1.260 \sim 1.655)$ & $<0.001^{*}$ \\
\hline Boarding status, N (\%) & $443(31.8)$ & $894(33.3)$ & 0.429 & & \\
\hline Key class, N (\%) & $645(46.3)$ & $1127(41.6)$ & $0.004^{*}$ & $1.128(0.980 \sim 1.299)$ & 0.093 \\
\hline Three generational household, N (\%) & $1070(76.8)$ & $2096(77.4)$ & 0.656 & & \\
\hline Single parent family, N (\%) & $221(14.4)$ & $228(8.4)$ & $<0.001^{*}$ & $1.870(1.524 \sim 2.294)$ & $<0.001^{*}$ \\
\hline Current caregivers, N (\%) & & & 0.797 & & \\
\hline Parents & $1160(83.3)$ & $2233(82.5)$ & & & \\
\hline Grandparents & $203(14.6)$ & $416(15.4)$ & & & \\
\hline Other relatives & $30(2.2)$ & $58(2.1)$ & & & \\
\hline Sibling children, N (\%) & $769(55.2)$ & $1512(55.9)$ & 0.691 & & \\
\hline Left behind types, N (\%) & & & 0.109 & & \\
\hline Un-left-behind & $933(67.0)$ & $1896(70.0)$ & & & \\
\hline Father absent & $94(6.7)$ & $149(5.5)$ & & & \\
\hline Mother absent & $42(3.0)$ & $62(2.30$ & & & \\
\hline Both absent & $324(23.3)$ & $600(22.2)$ & & & \\
\hline Father's education level, N (\%) & & & 0.265 & & \\
\hline Primary School and Below & $140(10.1)$ & $236(8.7)$ & & & \\
\hline Junior middle school & $427(32.1)$ & $915(33.8)$ & & & \\
\hline Senior middle school & $362(26.0)$ & $738(27.3)$ & & & \\
\hline College degree and above & $444(31.9)$ & $818(30.2)$ & & & \\
\hline Mother's education level, N (\%) & & & 0.665 & & \\
\hline Primary School and Below & $264(19.0)$ & $514(19.0)$ & & & \\
\hline Junior middle school & $387(27.8)$ & $800(29.6)$ & & & \\
\hline Senior middle school & $352(25.3)$ & $662(24.5)$ & & & \\
\hline College degree and above & $390(28.0)$ & $731(27.0)$ & & & \\
\hline Physical activity, N (\%) & $242(17.4)$ & $650(24.0)$ & $<0.001^{*}$ & $0.678(0.572 \sim 0.804)$ & $<0.001^{*}$ \\
\hline Birth order, N (\%) & & & 0.414 & & \\
\hline Only children & $624(44.8)$ & $1195(44.1)$ & & & \\
\hline Eldest children & $375(26.9)$ & $683(25.2)$ & & & \\
\hline Middle children & $116(8.3)$ & $250(9.2)$ & & & \\
\hline Youngest children & $278(20.0)$ & $579(21.4)$ & & & \\
\hline Having younger brother(s), N (\%) & $373(26.8)$ & $593(25.6)$ & 0.416 & & \\
\hline Having younger sister(s), N (\%) & $209(15.0)$ & $384(14.2)$ & 0.481 & & \\
\hline Having elder brother(s), N (\%) & $205(14.7)$ & $395(14.6)$ & 0.915 & & \\
\hline Having elder sister(s), N (\%) & $268(19.2)$ & $583(21.5)$ & 0.086 & $1.014(0.856 \sim 1.202)$ & 0.869 \\
\hline
\end{tabular}

$O R$ odds ratio, $\mathrm{Cl}$ confidence interval

*Statistically significant at $p<0.05$ level, two-sided

suicide attempts and psychiatric disorders in adolescence [26] and sibling bullying may be a significant driver for this phenomenon $[27,28]$. Contrary to previous survey, we mentioned that birth order was not significantly associated with high depressive symptomatology in both male and female adolescents. We suspect it because parents in China tend to favor the younger children, especially their youngest sons. The results of the present 
Table $\mathbf{2}$ Characteristics of male and female adolescents

\begin{tabular}{|c|c|c|c|}
\hline Variables & Male $(N=2326)$ & Female $(N=1774)$ & $P$ \\
\hline Rural areas, N (\%) & $1193(51.3)$ & $731(41.2)$ & $<0.001^{*}$ \\
\hline Age, y (IQR) & $13(13 \sim 14)$ & $13(13 \sim 14)$ & 0.707 \\
\hline Boarding status, N (\%) & $862(37.1)$ & $475(26.8)$ & $<0.001^{*}$ \\
\hline Key class, N (\%) & $931(40.0)$ & $841(47.4)$ & $<0.001^{*}$ \\
\hline Three generational household, N (\%) & $1795(77.2)$ & $1371(77.3)$ & 0.933 \\
\hline Single parent family, N (\%) & $264(11.3)$ & $165(9.3)$ & $0.034^{*}$ \\
\hline Current caregivers, N (\%) & & & $0.001^{*}$ \\
\hline Parents & $1883(81.0)$ & $1510(85.1)$ & \\
\hline Grandparents & $381(16.4)$ & $238(13.4)$ & \\
\hline Other relatives & $62(2.7)$ & $26(1.5)$ & \\
\hline Sibling children, N (\%) & $1280(55.0)$ & $1001(56.4)$ & 0.373 \\
\hline Left behind types, N (\%) & & & 0.151 \\
\hline Un-left-behind & $1576(67.8)$ & $1253(70.6)$ & \\
\hline Father absent & $139(6.0)$ & $104(5.9)$ & \\
\hline Mother absent & $57(2.9)$ & $47(2.6)$ & \\
\hline Both absent & $554(23.8)$ & $370(20.9)$ & \\
\hline Father's education level, N (\%) & & & $<0.001^{*}$ \\
\hline Primary School and Below & $233(10.0)$ & $143(8.1)$ & \\
\hline Junior middle school & $836(35.9)$ & $526(29.7)$ & \\
\hline Senior middle school & $608(26.1)$ & $492(27.7)$ & \\
\hline College degree and above & $649(27.9)$ & $613(34.6)$ & \\
\hline Mother's education level, N (\%) & & & $<0.001^{*}$ \\
\hline Primary School and Below & $486(20.9)$ & $292(16.5)$ & \\
\hline Junior middle school & $707(30.4)$ & $480(27.1)$ & \\
\hline Senior middle school & $552(23.7)$ & $462(26.0)$ & \\
\hline College degree and above & $581(25.0)$ & $540(30.4)$ & \\
\hline Physical activity, N, (\%) & $616(26.5)$ & $276(15.6)$ & $<0.001^{*}$ \\
\hline Birth order, N, (\%) & & & $<0.001$ \\
\hline Only children & $1046(45.0)$ & $773(43.6)$ & \\
\hline Eldest children & $444(19.1)$ & $614(31.6)$ & \\
\hline Middle children & $235(10.1)$ & $131(7.4)$ & \\
\hline Youngest children & $601(25.8)$ & $256(14.4)$ & \\
\hline Having younger brother(s), N, (\%) & $466(20.0)$ & $600(33.8)$ & $<0.001^{*}$ \\
\hline Having younger sister(s), N, (\%) & $358(15.4)$ & $235(13.2)$ & 0.053 \\
\hline Having elder brother(s), N, (\%) & $355(15.3)$ & $254(13.8)$ & 0.193 \\
\hline Having elder sister(s), N, (\%) & $640(27.5)$ & $211(11.9)$ & $<0.001^{*}$ \\
\hline High depressive symptomatology, N, (\%) & $703(30.2)$ & $690(38.9)$ & $<0.001$ \\
\hline
\end{tabular}

*Statistically significant at $p<0.05$ level, two-sided

study also support this idea, we can see that male adolescents were obviously more than female adolescents among these youngest children $(N=857,70.1 \%$ VS. $29.9 \%)$.

Recent years, the physical psychological problems of left-behind children in China have aroused extensive concerns. Left-behind children have more mental and behavioral problems compared with general population of children [29, 30]. Meanwhile, some studies also present different results [31]. In China, the long-term impacts of traditional concepts lead parents to focus almost exclusively on their children's academic performance rather than on psychological well-being [32]. Adolescents living with parents might suffering physical 
Table 3 Univariate and multivariate logistic regression analysis for high depressive symptomatology in male adolescents

\begin{tabular}{|c|c|c|c|c|}
\hline \multirow[t]{2}{*}{ Correlates } & \multicolumn{2}{|c|}{ Univariate analysis } & \multicolumn{2}{|c|}{ Multivariate analysis } \\
\hline & $P$ & Crude OR $(95 \% \mathrm{Cl})$ & $P$ & Adjusted OR $(95 \% \mathrm{Cl})$ \\
\hline Rural areas & 0.439 & $0.932(0.781 \sim 1.113)$ & & \\
\hline Age & $0.009^{*}$ & $1.129(1.031 \sim 1.236)$ & $0.009^{*}$ & $1.145(1.034 \sim 1.266)$ \\
\hline Boarding status & 0.328 & $1.095(0.913 \sim 1.315)$ & & \\
\hline Key class & $0.030^{*}$ & $1.220(1.020 \sim 1.461)$ & 0.058 & $1.206(0.994 \sim 1.464)$ \\
\hline Three generational household & 0.627 & $0.949(0.770 \sim 1.171)$ & & \\
\hline Single parent family & $<0.001^{*}$ & $1.801(1.386 \sim 2.342)$ & $<0.001^{*}$ & $1.734(1.327 \sim 2.265)$ \\
\hline Current caregivers & 0.783 & & & \\
\hline Parents & & 1.00 & & \\
\hline Grandparents & 0.546 & $0.928(0.728 \sim 1.182)$ & & \\
\hline Other relatives & 0.758 & $1.089(0.634 \sim 1.871)$ & & \\
\hline Sibling children & 0.938 & $0.993(0.831 \sim 1.186$ & & \\
\hline Left behind types & 0.072 & & 0.071 & \\
\hline Un-left-behind & & 1.00 & & 1.00 \\
\hline Father absent & $0.035^{*}$ & $1.473(1.027 \sim 2.112)$ & 0.053 & $1.436(0.996 \sim 2.071)$ \\
\hline Mother absent & 0.115 & $1.549(0.899 \sim 2.669)$ & 0.181 & $1.462(0.838 \sim 2.550)$ \\
\hline Both absent & 0.260 & $1.128(0.915 \sim 1.392)$ & 0.077 & $1.225(0.978 \sim 1.534)$ \\
\hline Father's education level & 0.883 & & & \\
\hline Primary School and Below & & 1.00 & & \\
\hline Junior middle school & 0.824 & $0.965(0.704 \sim 1.322)$ & & \\
\hline Senior middle school & 0.611 & $0.918(0.661 \sim 1.275)$ & & \\
\hline College degree and above & 0.950 & $1.011(0.731 \sim 1.397)$ & & \\
\hline Mother's education level & 0.928 & & & \\
\hline Primary School and Below & & 1.00 & & \\
\hline Junior middle school & 0.942 & $0.991(0.770 \sim 1.275)$ & & \\
\hline Senior middle school & 0.907 & $0.984(0.754 \sim 1.285)$ & & \\
\hline College degree and above & 0.651 & $1.062(0.818 \sim 1.380)$ & & \\
\hline Physical activity & $<0.001^{*}$ & $0.666(0.539 \sim 0.822)$ & $<0.001^{*}$ & $0.632(0.509 \sim 0.783)$ \\
\hline Birth order & 964 & & & \\
\hline Only children & & 1.00 & & \\
\hline Eldest children & 0.692 & $0.952(0.747 \sim 1.214)$ & & \\
\hline Middle children & 0.978 & $0.996(0.732 \sim 1.355)$ & & \\
\hline Youngest children & 0.840 & $1.023(0.823 \sim 1.271)$ & & \\
\hline Having younger brother(s) & 0.749 & $0.964(0.772 \sim 1.204)$ & & \\
\hline Having younger sister(s) & 0.635 & $1.061(0.832 \sim 1.353)$ & & \\
\hline Having elder brother(s) & 0.734 & $1.043(0.817 \sim 1.333)$ & & \\
\hline Having elder sister(s) & 0.954 & $1.006(0.825 \sim 1.226)$ & & \\
\hline
\end{tabular}

OR odds ratio, $\mathrm{Cl}$ confidence interval

*Statistically significant at $p<0.05$ level, two-sided

and verbal punishment when they didn't study hard, which increased the risk of high depressive symptomatology. Some researchers have also found that adolescents whose father came back home more frequently displayed poorer health-related quality of life [33]. Thus, the current view on relationship between left-behind and depressive symptoms remains controversial. In this study, left behind status were not significantly related to high depressive symptomatology in both mela and female adolescents.

This study shown that the prevalence of high depressive symptomatology in female adolescents was significantly 
Table 4 Univariate and multivariate logistic regression analysis for high depressive symptomatology in female adolescents

\begin{tabular}{|c|c|c|c|c|}
\hline \multirow[t]{2}{*}{ Correlates } & \multicolumn{2}{|c|}{ Univariate analysis } & \multicolumn{2}{|c|}{ Multivariate analysis } \\
\hline & $P$ & Crude OR $(95 \% \mathrm{Cl})$ & $P$ & Adjusted OR $(95 \% \mathrm{Cl})$ \\
\hline Rural areas & 0.307 & $0.904(0.744 \sim 1.098)$ & & \\
\hline Age & $0.003^{*}$ & $1.172(1.057 \sim 1.300)$ & $0.001^{*}$ & $1.201(1.076 \sim 1.341)$ \\
\hline Boarding status & 0.161 & $0.856(0.689 \sim 1.064)$ & & \\
\hline Key class & 0.209 & $1.130(0.934 \sim 1.369)$ & & \\
\hline Three generational household & 0.884 & $0.983(0.783 \sim 1.235)$ & & \\
\hline Single parent family & $<0.001^{*}$ & $2.018(1.461 \sim 2.786)$ & $<0.001^{*}$ & $2.004(1.448 \sim 2.772)$ \\
\hline Current caregivers & 0.997 & & & \\
\hline Parents & & 1.00 & & \\
\hline Grandparents & 0.953 & $1.009(0.762 \sim 1.335)$ & & \\
\hline Other relatives & 0.966 & $1.089(0.634 \sim 1.871)$ & & \\
\hline Sibling children & 0.533 & $0.941(0.776 \sim 1.140)$ & & \\
\hline Left behind types & 0.783 & & & \\
\hline Un-left-behind & & 1.00 & & \\
\hline Father absent & 0.652 & $1.098(0.730 \sim 1.652)$ & & \\
\hline Mother absent & 0.542 & $1.201(0.666 \sim 2.165)$ & & \\
\hline Both absent & 0.407 & $1.105(0.872 \sim 1.401)$ & & \\
\hline Father's education level & 0.131 & & 0.74 & \\
\hline Primary School and Below & & 1.00 & & 1.00 \\
\hline Junior middle school & $0.023^{*}$ & $0.650(0.448 \sim 0.943)$ & $0.021^{*}$ & $0.641(0.439 \sim 0.934)$ \\
\hline Senior middle school & $0.033^{*}$ & $0.665(0.457 \sim 0.968)$ & $0.010^{*}$ & $0.603(0.410 \sim 0.888)$ \\
\hline College degree and above & 0.078 & $0.719(0.499 \sim 1.037)$ & $0.021^{*}$ & $0.639(0.437 \sim 0.936)$ \\
\hline Mother's education level & 0.584 & & & \\
\hline Primary School and Below & & 1.00 & & \\
\hline Junior middle school & 0.299 & $0.854(0.633 \sim 1.151)$ & & \\
\hline Senior middle school & 0.939 & $1.012(0.751 \sim 1.364)$ & & \\
\hline College degree and above & 0.594 & $0.924(0.691 \sim 1.236)$ & & \\
\hline Physical activity & $<0.054^{*}$ & $0.767(0.585 \sim 1.005)$ & 0.073 & $0.778(0.592 \sim 1.023)$ \\
\hline Birth order & 0.508 & & & \\
\hline Only children & & 1.00 & & \\
\hline Eldest children & 0.944 & $1.008(0.812 \sim 1.251)$ & & \\
\hline Middle children & 0.245 & $0.794(0.539 \sim 1.171)$ & & \\
\hline Youngest children & 0.335 & $0.866(0.646 \sim 1.160)$ & & \\
\hline Having younger brother(s) & 0.867 & $1.017(0.832 \sim 1.245)$ & & \\
\hline Having younger sister(s) & 0.422 & $1.121(0.848 \sim 1.483)$ & & \\
\hline Having elder brother(s) & 0.967 & $0.994(0.754 \sim 1.311)$ & & \\
\hline Having elder sister(s) & 0.225 & $0.830(0.615 \sim 1.121)$ & & \\
\hline
\end{tabular}

$O R$ odds ratio, $\mathrm{Cl}$ confidence interval

*Statistically significant at $p<0.05$ level, two-sided

higher than male adolescents. Further subgroup analysis revealed that age, single parent family and fathers' education background were associated with high depressive symptomatology in female adolescents, fathers' education background was only associated with high depressive symptomatology in female adolescents, but not with male adolescents.

Previous research has suggested that females, whether adults or adolescents, were more likely to suffer depression, and this gender difference emerges from adolescence 
[34]. As shown in Fig. 1, in this study, the prevalence increased with age in both female adolescents and male adolescents (female $34.3 \%$ vs. male $24.2 \%$ in $11-12$ group, $39.1 \%$ vs. $30.4 \%$ in $13-14$ group, $44.4 \%$ vs. $35.9 \%$ in $15-16$ group, respectively), and the gender difference existed in each age group. Previous studies suggested that the gender difference of depression in adolescents emerges between ages 12 and 15, while females experience increases in depression symptoms in early adolescence and males experience increases in depression symptoms in later adolescence [35-38]. A recent longitudinal study indicated that girls' diagnostic rates of depression increased from ages 11 through 14, whereas boys' diagnostic rates of depression increased from ages 15 through 18 [39]. However, all these researches were conducted in United States. The national adolescent sample in China showed that prevalence of depressive symptoms in both boys and girls had increased from 7th to 9th grade [40]. This may be due to the cultural differences between China and United States. Asian cultures often value modesty and selfeffacement as cultural virtues and inhibit the expression of positive emotion [41, 42], which would also lead to differences in prevalence between male and female adolescents. A cross-cultural comparative study found that after controlling for level of income, age, education, and previous chemotherapy, there was a significant cultural difference in the positive affect subscale between Chinese and US female [43]. These results are consistent with other crosscultural comparisons of CES-D scores from healthy community Asian populations including South Koreans, Japanese, and Chinese, which have reported that scores on positive affect components are lower, and can lead to higher depressive symptom scores [41, 42, 44-46].

Subgroup analysis shows that female adolescents whose father with primary school education or below were more susceptible to high depressive symptomatology (Table 4). From Fig. 2 we can see that prevalence of high depressive symptomatology in girls whose father with primary school education or below was much higher than any other girls, whereas prevalence of high depressive symptomatology in male adolescents has remained relatively unaffected by fathers' education level.

Traditional preferences for sons are deep-rooted in rural areas of China, especially in low educated communities [47-50]. According to the Global Gender Gap Report 2018, China ranked dead last among 149 countries in terms of "sex ratio at birth". This is the result of many factors, such as the Confucian cultural tradition, the socioeconomic system, and gender ideology. In the context of traditional patriarchal, patrilineal and patriarchal systems, sons are considered to have unique value, as they inherit the family name and property and represent an economic value premium to the family and parents $[48$, 50-52]. Under the one-child policy in China, a family with strong preference for sons may be trying to conduct gender selection [49]. Since the universal two-child policy was finally introduced in 2016, the families with an

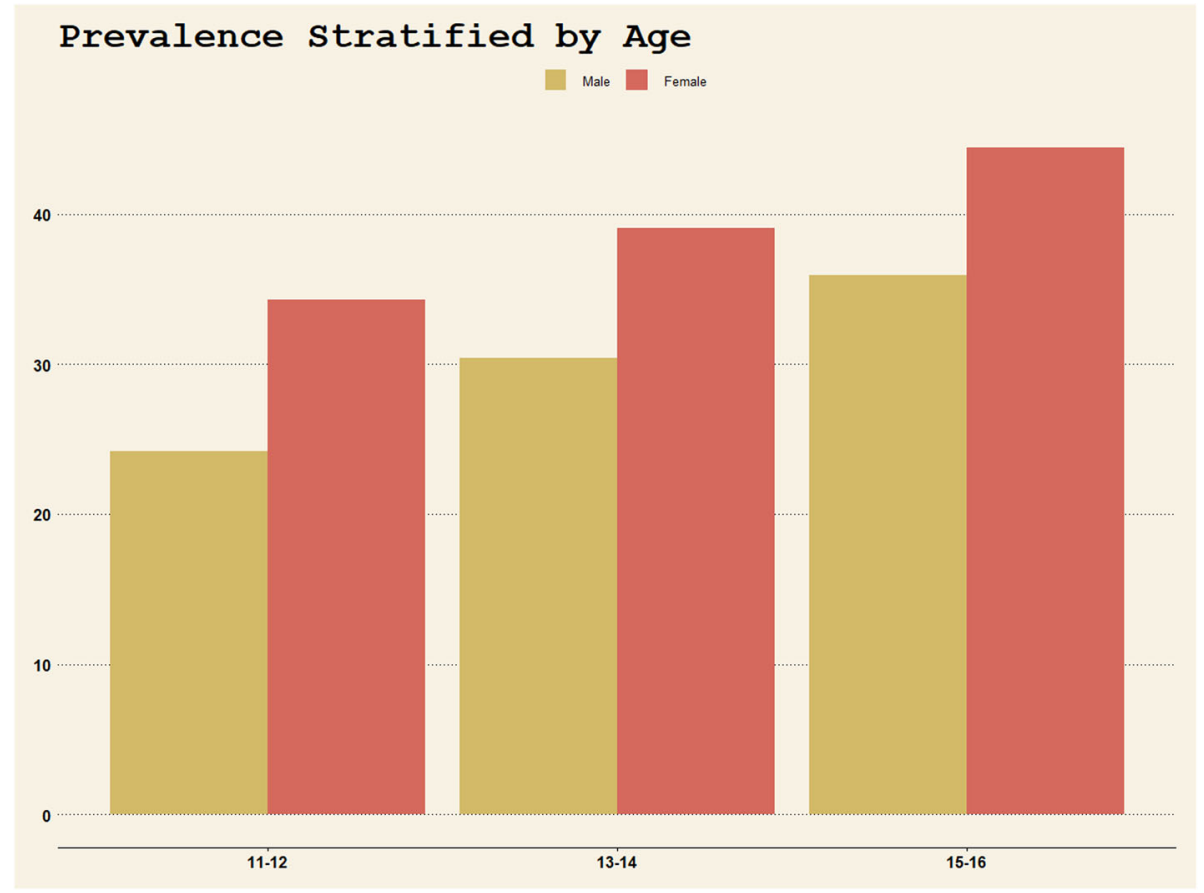

Fig. 1 Prevalence stratified by age 


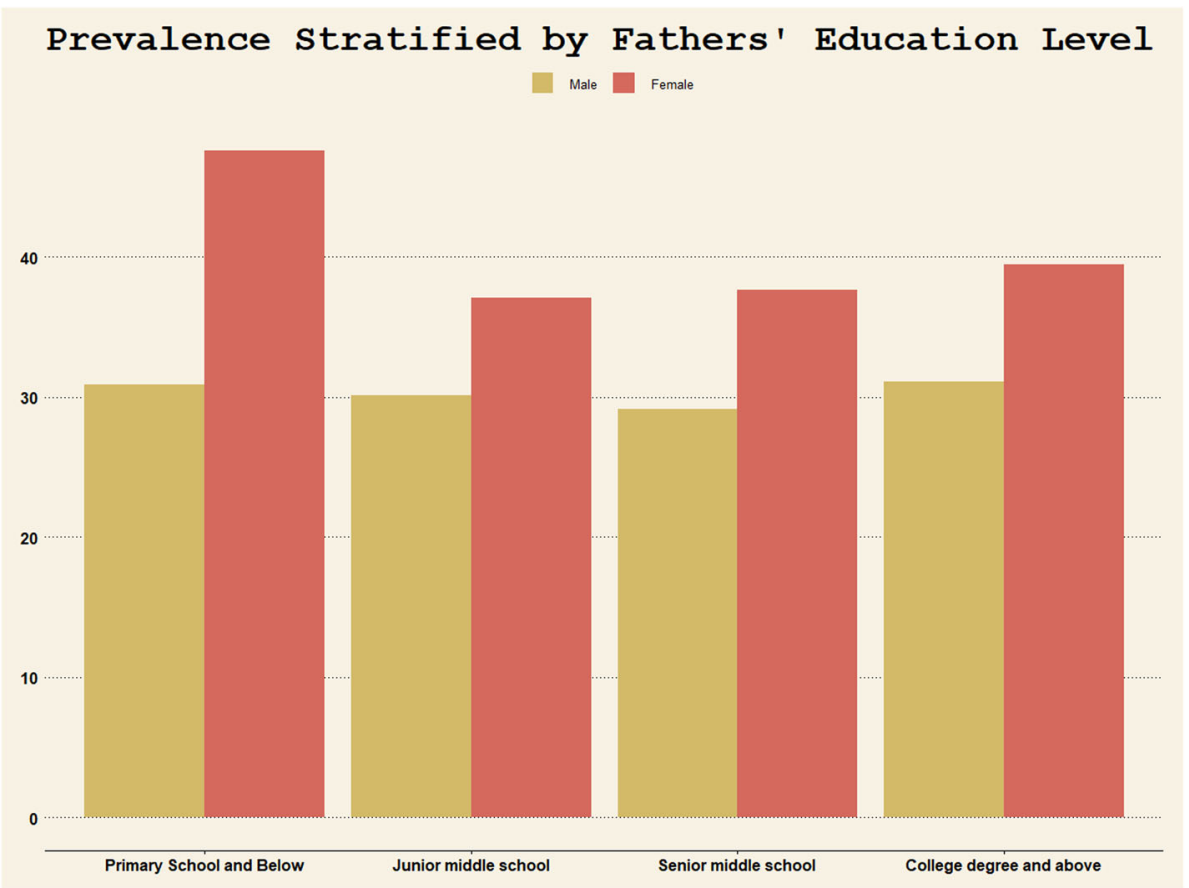

Fig. 2 Prevalence stratified by fathers' education level

only daughter are more inclined to have another son [49]. Under such circumstances, female adolescents often face inequity in education and constrained by low expectations. Our study also confirms this conjecture to some extent. From the comparison of psycho-social characteristics between boys and girls (Table 2) we can see that proportion of male adolescents in rural areas was significantly higher than female adolescents ( 51.3 vs. $41.2 \%)$. In addition, significantly more girls have younger brother at home (33.8 vs. $20.0 \%)$ and less girls have older sister (11.9 vs. 27.5\%). These unusual proportions could show that son preference is still widespread in China, especially in rural areas. Meanwhile, we observed that more female adolescents' parents, both fathers and mothers, have higher education level. The role that fathers' educational level plays in depressive symptoms may be explained by previous studies showing that fathers with a higher educational level tend to be warmer and more communicative with their children and to possess better emotional skills $[53,54]$. Fathers with primary school education or below usually unable to provide favorable education and economic conditions to their daughters, which may also increase the prevalence of high depressive symptomatology. A study reported that father's education level was positively associated with girl's well-being in rural China [55], indicating that better educated fathers may value their daughters' development more. Moreover, growing body of researches indicated that parental preference for sons might reduce under the modern outlook of fertility in urban areas but remained strong among rural-urban migrants $[48,49$, 56], which means it's the higher education levels of parents, not living areas, reduce the preference for sons.

\section{Strengths and limitations}

To the best of our knowledge, this is the first large sample cross-sectional study to explore the different related factors of high depressive symptomatology in female and male adolescents in central China. The findings of this study provide etiological clues to gender difference in adolescent depression. At the same time, there are some limitations in this study. First, in this study, some variables are not included because they don't have clear cause-and-effect relationship with depression or not objective enough, such as family financial situation, academic achievement, pressure of interpersonal relationship, etc. Second, our sample was confined to Hubei province, the conclusion needs further verification in other regions of China. Third, the CES-D scale was a brief instrument to measure the burden of depression, not able to provide a clinical depression diagnosis.

\section{Conclusion}

Our study focuses on psycho-social factors related to female adolescents with high depressive symptomatology and gender differences in depressive symptoms among Chinese adolescents. We found that adolescents from single parent family, adolescents with older age and 
adolescents with less physical activity were more susceptible to high depressive symptomatology. However, fathers' education background was only associated with high depressive symptomatology in female adolescents. Female adolescents whose father with primary school education or below deserves more attention. In China, especially in low educated communities, son preferences still widespread and popularization of rural compulsory education is the priority of China's educational development. Furthermore, it is imperative to confirm these findings through nationally representative study.

\section{Abbreviations \\ OR: Odds ratio; Cl: Confidence interval; WHO: World Health Organization; CES-D: Center for Epidemiology Studies Depression Scale; PA: Physical activity; LBC: Left-behind children; IQR: Interquartile ranges}

\section{Acknowledgments}

The authors thank the research personnel who assisted with data collection. We would like to acknowledge all participants of this project and investigators for collecting data. Also, we wish to thank local education bureau for supporting this study.

\section{Authors' contributions}

Concept and design: Y.X., Z.Z., J.M., C.Z.; Acquisition, analysis, and interpretation of data: W.S., G.L., X.Z., Y.W.; Drafting of the manuscript: W.S., Y.W., X.Z., Y.L., Z.Z.; Critical revision of the manuscript: Y.X., J.M., Y.C., Z.Z.; Statistical analysis: W.S., C.P., X.Q., J.M.; The author(s) read and approved the final manuscript.

\section{Funding}

This work was financially supported by the National Key R\&D Program of China [grant number 2017YFC1310000], the Fundamental Research Funds for the Central Universities [grant number 2018KFYXMPT015] and Hubei Technological Innovation Special Fund (CN) [grant number 2019ACA132]. The funders had no role in study design, data collection and analysis, decision to publish, or preparation of the manuscript. The funders had no role in study design, data collection and analysis, decision to publish, or preparation of the manuscript.

\section{Availability of data and materials}

The de-identified database used in the current study are available from the corresponding author on reasonable request.

\section{Declarations}

\section{Ethics approval and consent to participate}

The study was approved by the Ethics Committee of Tongji Medical College, Huazhong University of Science and Technology. All procedures performed in this study involving human participants were in accordance with the 1964 Helsinki declaration and its later amendments. The written informed consent was obtained from a parent or guardian for participants under 18 years old. Participants were informed that the survey was totally voluntary. The subjects' confidentiality was protected by ensuring that the data were addressed in anonymous mode with personal information appropriately deidentified. All the answers were collected by close-ended categorical items at the beginning of the questionnaire.

\section{Consent for publication}

Not applicable.

\section{Competing interests}

All authors declare that they have no conflicts of interest.

\section{Author details}

'Department of Neurology, Tongji Hospital, Tongji Medical College, Huazhong University of Science and Technology, No.1095 Jiefang Avenue, Wuhan 430030, China. ${ }^{2}$ Department of Neurology, Wuhan First Hospital,
No.215 Zhongshan Avenue, Wuhan 430030, Hubei, China. ${ }^{3}$ The Solomon H. Snyder Department of Neuroscience, Johns Hopkins University School of Medicine, Baltimore, MD 21205, USA. ${ }^{4}$ Department of Plastic surgery, Tongji Hospital, Tongji Medical College, Huazhong University of Science and

Technology, No.1095 Jiefang Avenue, Wuhan 430030, China.

Received: 10 November 2020 Accepted: 11 March 2021

Published online: 26 March 2021

\section{References}

1. Barker MM, Beresford B, Bland M, Fraser LK. Prevalence and incidence of anxiety and depression among children, adolescents, and young adults with life-limiting conditions: a systematic review and meta-analysis. JAMA Pediatr. 2019;173(9):835-44. https://doi.org/10.1001/jamapediatrics.2019.1 712.

2. Dwyer JB, Aftab A, Widge A, Rodriguez Cl, Carpenter LL, Nemeroff CB, et al. Hormonal treatments for major depressive disorder: state of the art. Am J Psychiatry. 2020;177(8):686-705 appiajp202019080848.

3. Chi X, Liu X, Huang Q, Huang L, Zhang P, Chen X. Depression in Chinese adolescents: prevalence, changes, and social-demographic correlates. J Affect Disord. 2020;274:1191-200.

4. Jane Costello E, Erkanli A, Angold A. Is there an epidemic of child or adolescent depression? J Child Psychol Psychiatry. 2006;47(12):1263-71.

5. Kessler RC, Berglund P, Demler O, Jin R, Merikangas KR, Walters EE. Lifetime prevalence and age-of-onset distributions of DSM-IV disorders in the National Comorbidity Survey Replication. Arch Gen Psychiatry. 2005;62(6): 593-602. https://doi.org/10.1001/archpsyc.62.6.593.

6. Kim-Cohen J, Caspi A, Moffitt TE, Harrington H, Milne BJ, Poulton R. Prior juvenile diagnoses in adults with mental disorder: developmental followback of a prospective-longitudinal cohort. Arch Gen Psychiatry. 2003;60(7): 709-17. https://doi.org/10.1001/archpsyc.60.7.709.

7. Zhong BL, Ding J, Chen HH, Li Y, Xu HM, Tong J, Wang AQ, Tang GZ, Zhu JS, Yang DQ, Liu B, Wang Q, Cheng WF, Yin E, Xu MJ, Zhang T, Hu TM, Feng XW, Li H, Dan TQ, Cheng GM, Zhang JF, Li HJ, Zhu JH. Depressive disorders among children in the transforming China: an epidemiological survey of prevalence, correlates, and service use. Depress Anxiety. 2013;30(9):881-92. https://doi.org/10.1002/da.22109.

8. Li M, Xue H, Wang W, Wang Y. Parental expectations and child screen and academic sedentary behaviors in China. Am J Prev Med. 2017;52(5):680-9. https://doi.org/10.1016/j.amepre.2016.12.006.

9. Ying L, Zhou H, Yu S, Chen C, Jia X, Wang Y, et al. Parent-child communication and self-esteem mediate the relationship between interparental conflict and children's depressive symptoms. Child Care Health Dev. 2018;44(6):908-15.

10. Wang L, Feng Z, Yang G, Yang Y, Wang K, Dai Q, Zhao M, Hu C, Zhang R, Liu K, Guang Y, Xia F. Depressive symptoms among children and adolescents in western China: an epidemiological survey of prevalence and correlates. Psychiatry Res. 2016;246:267-74. https://doi.org/10.1016/j. psychres.2016.09.050.

11. Li G, Mei J, You J, Miao J, Song X, Sun W, Lan Y, Qiu X, Zhu Z. Sociodemographic characteristics associated with adolescent depression in urban and rural areas of Hubei province: a cross-sectional analysis. BMC Psychiatry. 2019;19(1):386. https://doi.org/10.1186/s12888-019-2380-4.

12. Chi X, Liu X, Huang $Q$, Huang L, Zhang $P$, Chen $X$. Depressive symptoms among junior high school students in southern China: prevalence, changes, and psychosocial correlates. J Affect Disord. 2020;274:1191-200. https://doi. org/10.1016/j.jad.2020.05.034.

13. Luo D, Yan X, Xu R, Zhang J, Shi X, Ma J, Song Y, Patton GC, Sawyer SM. Chinese trends in adolescent marriage and fertility between 1990 and 2015: a systematic synthesis of national and subnational population data. Lancet Glob Health. 2020;8(7):e954-e64. https://doi.org/10.1016/S2214-109X(20)3 0130-3.

14. Naghavi M. Global, regional, and national burden of suicide mortality 1990 to 2016: systematic analysis for the Global Burden of Disease Study 2016. BMJ. 2019;364:194.

15. Cheng ST, Chan AC. The center for epidemiologic studies depression scale in older Chinese: thresholds for long and short forms. Int J Geriatr Psychiatry. 2005;20(5):465-70. https://doi.org/10.1002/gps.1314.

16. Zhang Y, Ting RZ, Lam MH, Lam SP, Yeung RO, Nan H, et al. Measuring depression with CES-D in Chinese patients with type 2 diabetes: the validity 
and its comparison to PHQ-9. BMC Psychiatry. 2015;15(1):198. https://doi. org/10.1186/s12888-015-0580-0.

17. Stahl D, Sum CF, Lum SS, Liow PH, Chan YH, Verma S, Chua HC, Chong SA Screening for depressive symptoms: validation of the center for epidemiologic studies depression scale (CES-D) in a multiethnic group of patients with diabetes in Singapore. Diabetes Care. 2008;31(6):1118-9. https://doi.org/10.2337/dc07-2019.

18. Yang HJ, Soong WT, Kuo PH, Chang HL, Chen WJ. Using the CES-D in a two-phase survey for depressive disorders among nonreferred adolescents in Taipei: a stratum-specific likelihood ratio analysis. J Affect Disord. 2004; 82(3):419-30.

19. Vilagut $G$, Forero CG, Barbaglia G, Alonso J. Screening for depression in the general population with the Center for Epidemiologic Studies Depression (CES-D): a systematic review with meta-analysis. PLoS One. 2016;11(5): e0155431. https://doi.org/10.1371/journal.pone.0155431.

20. Zhou M, Zhang G, Rozelle S, Kenny K, Xue H. Depressive symptoms of Chinese children: prevalence and correlated factors among subgroups. Int J Environ Res Public Health. 2018;15(2):283.

21. Verhoeven M, Sawyer MG, Spence SH. The factorial invariance of the CES-D during adolescence: are symptom profiles for depression stable across gender and time? J Adolesc. 2013;36(1):181-90. https://doi.org/10.1016/j.a dolescence.2012.10.007.

22. Chi X, Huang L, Wang J, Zhang P. The prevalence and socio-demographic correlates of depressive symptoms in early adolescents in china: differences in only child and non-only child groups. Int J Environ Res Public Health. 2020;17(2):438

23. Wang PW, Lin HC, Yeh YC, Liu TL, Yen CF. The relation of substance use with different levels of depressive symptoms and the moderating effect of sex and age in Taiwanese adolescents. Compr Psychiatry. 2012;53(7):101320. https://doi.org/10.1016/j.comppsych.2012.02.010.

24. Lee D-E, Kwon SW. Community-level factors and adolescent depression in South Korea: socioeconomic composition, education environment, and community wellbeing. Child Indic Res. 2015;8(2):459-70. https://doi.org/10.1 007/s12187-014-9259-1.

25. He B, Fan J, Liu N, Li H, Wang Y, Williams J, Wong K. Depression risk of 'leftbehind children' in rural China. Psychiatry Res. 2012;200(2-3):306-12. https:// doi.org/10.1016/j.psychres.2012.04.001.

26. Easey KE, Mars B, Pearson R, Heron J, Gunnell D. Association of birth order with adolescent mental health and suicide attempts: a population-based longitudinal study. Eur Child Adolesc Psychiatry. 2019;28(8):1079-86. https:// doi.org/10.1007/s00787-018-1266-1.

27. Bowes $L$, Wolke D, Joinson C, Lereya ST, Lewis G. Sibling bullying and risk of depression, anxiety, and self-harm: a prospective cohort study. Pediatrics. 2014;134(4):e1032-9. https://doi.org/10.1542/peds.2014-0832.

28. Dantchev S, Zammit S, Wolke D. Sibling bullying in middle childhood and psychotic disorder at 18 years: a prospective cohort study. Psychol Med. 2018;48(14):2321-8. https://doi.org/10.1017/\$0033291717003841.

29. Qu X, Wang X, Huang X, Ashish KC, Yang Y, Huang Y, Chen C, Gao Y, Wang $Y$, Zhou H. Socio-emotional challenges and development of children left behind by migrant mothers. J Glob Health. 2020;10(1):010806. https://doi. org/10.7189/jogh.10.010806.

30. Guan H, Wang H, Huang J, Du K, Zhao J, Boswell M, et al. Health seeking behavior among rural left-behind children: evidence from Shaanxi and Gansu Provinces in China. Int J Environ Res Public Health. 2018;15(5):883.

31. Shen M, Gao J, Liang Z, Wang Y, Du Y, Stallones L. Parental migration patterns and risk of depression and anxiety disorder among rural children aged 10-18 years in China: a cross-sectional study. BMJ Open. 2015;5(12): e007802. https://doi.org/10.1136/bmjopen-2015-007802.

32. Aldinger $C$, Zhang XW, Liu LQ, Pan XD, Yu SH, Jones J, Kass J. Changes in attitudes, knowledge and behavior associated with implementing a comprehensive school health program in a province of China. Health Educ Res. 2008;23(6):1049-67. https://doi.org/10.1093/her/cyn022.

33. Huang $Y$, Zhong $X N$, Li QY, Xu D, Zhang XL, Feng C, Yang GX, Bo YY, Deng $B$. Health-related quality of life of the rural-China left-behind children or adolescents and influential factors: a cross-sectional study. Health Qual Life Outcomes. 2015;13(1):29. https://doi.org/10.1186/s12 955-015-0220-x

34. Nolen-Hoeksema S, Girgus JS. The emergence of gender differences in depression during adolescence. Psychol Bull. 1994;115(3):424-43. https://doi. org/10.1037/0033-2909.115.3.424
35. Ge X, Conger RD, Elder GH Jr. Pubertal transition, stressful life events, and the emergence of gender differences in adolescent depressive symptoms. Dev Psychol. 2001;37(3):404-17. https://doi.org/10.1037/0012-1649.37.3.404.

36. Hankin BL, Abramson LY, Moffitt TE, Silva PA, McGee R, Angell KE. Development of depression from preadolescence to young adulthood: emerging gender differences in a 10-year longitudinal study. J Abnorm Psychol. 1998;107(1):128-40. https://doi.org/10.1037/0021-843X.107.1.128.

37. Marmorstein NR. Longitudinal associations between alcohol problems and depressive symptoms: early adolescence through early adulthood. Alcohol Clin Exp Res. 2009;33(1):49-59. https://doi.org/10.1111/j.1530-0277.2008.0081 $0 . x$.

38. Avenevoli S, Swendsen J, He JP, Burstein M, Merikangas KR. Major depression in the national comorbidity survey-adolescent supplement: prevalence, correlates, and treatment. J Am Acad Child Adolesc Psychiatry. 2015:54(1):37-44.e2.

39. Salk RH, Petersen JL, Abramson LY, Hyde JS. The contemporary face of gender differences and similarities in depression throughout adolescence: development and chronicity. J Affect Disord. 2016;205:28-35. https://doi. org/10.1016/j.jad.2016.03.071.

40. Ma L, Gao L, Chiu DT, Ding Y, Wang W, Wang Y. Depressive symptoms prevalence, associated family factors, and gender differences: a national cohort study of middle school students in China. J Affect Disord. 2020;274 545-52. https://doi.org/10.1016/j.jad.2020.05.128.

41. Iwata N, Buka S. Race/ethnicity and depressive symptoms: a cross-cultural/ ethnic comparison among university students in East Asia, North and South America. Soc Sci Med. 2002;55(12):2243-52.

42. Jang Y, Kim G, Chiriboga D. Acculturation and manifestation of depressive symptoms among Korean-American older adults. Aging Ment Health. 2005; 9(6):500-7. https://doi.org/10.1080/13607860500193021.

43. Milbury K, Kavanagh A, Meng Z, Chen Z, Chandwani KD, Garcia K, Perkins GH, McQuade J, Raghuram NV, Nagarathna R, Liao Z, Nagendra HR, Chen J, Guo X, Liu L, Arun B, Cohen L. Depressive symptoms and positive affect in Chinese and United States breast cancer survivors: a cross-cultural comparison. Support Care Cancer. 2017;25(7):2103-9. https://doi.org/10.1 007/s00520-017-3612-0.

44. Iwata N, Higuchi HR. Responses of Japanese and American university students to the STAl items that assess the presence or absence of anxiety. J Pers Assess. 2000;74(1):48-62. https://doi.org/10.1207/S15327752JPA740104.

45. Jang Y, Kwag KH, Chiriboga DA. Not saying I am happy does not mean I am not: cultural influences on responses to positive affect items in the CESD. J Gerontol Ser B Psychol Sci Soc Sci. 2010;65(6):684-90.

46. Kanazawa A, White PM, Hampson SE. Ethnic variation in depressive symptoms in a community sample in Hawaii. Cult Divers Ethn Minor Psychol. 2007;13(1):35-44. https://doi.org/10.1037/1099-9809.13.1.35.

47. Dong H, Manfredini M, Kurosu S, Yang W, Lee JZ. Kin and birth order effects on male child mortality: three east Asian populations, 1716-1945(). Evol Hum Behav. 2017;38(2):208-16. https://doi.org/10.1016/j.evolhumbehav.201 6.10.001.

48. Das Gupta M, Zhenghua J, Bohua L, Zhenming X, Chung W, Hwa-Ok B. Why is son preference so persistent in East and South Asia? A cross-country study of China, India and the Republic of Korea. J Dev Stud. 2003:40(2):15387. https://doi.org/10.1080/00220380412331293807.

49. Wang $X$, Nie W, Liu P. Son preference and the reproductive behavior of rural-urban migrant women of childbearing Age in China: empirical evidence from a cross-sectional data. Int J Environ Res Public Health. 2020; 17(9):3221.

50. Tilt B, Li X, Schmitt EA. Fertility trends, sex ratios, and son preference among Han and minority households in rural China. Asian Anthropol. 2019;18(2): 110-28. https://doi.org/10.1080/1683478X.2019.1588199.

51. Larsen U, Chung W, Gupta MD. Fertility and son preference in Korea. Popul Stud. 1998;52(3):317-25. https://doi.org/10.1080/0032472031000150496.

52. Chaudhuri $\mathrm{S}$. The desire for sons and excess fertility: a household-level analysis of parity progression in India. Int Perspect Sex Reprod Health. 2012; 38(04):178-86. https://doi.org/10.1363/3817812.

53. Dornbusch SM, Ritter PL, Leiderman PH, Roberts DF, Fraleigh MJ. The relation of parenting style to adolescent school performance. Child Dev. 1987;58(5):1244-57. https://doi.org/10.2307/1130618.

54. Romero NAR, Guajardo JG, Aguirre FAR, Sánchez AM, Gallego NG. Inteligencia emocional en la mejora de los estilos educativos de padres con hijos en edad escolar. Salud Soc. 2012;3(3):283-91. https://doi.org/10.22199/ S07187475.2012.0003.00004. 
55. Wen M, Lin D. Child development in rural China: children left behind by their migrant parents and children of nonmigrant families. Child Dev. 2012; 83(1):120-36. https://doi.org/10.1111/j.1467-8624.2011.01698.x.

56. Liao QZ, Cao GZ, Tao R. A study on the fertility and son preferences of China's migrants: empirical evidence from 12 cities. Popul Dev. 2012;18:2-12.

\section{Publisher's Note}

Springer Nature remains neutral with regard to jurisdictional claims in published maps and institutional affiliations.

Ready to submit your research? Choose BMC and benefit from:

- fast, convenient online submission

- thorough peer review by experienced researchers in your field

- rapid publication on acceptance

- support for research data, including large and complex data types

- gold Open Access which fosters wider collaboration and increased citations

- maximum visibility for your research: over $100 \mathrm{M}$ website views per year

At $\mathrm{BMC}$, research is always in progress.

Learn more biomedcentral.com/submissions 